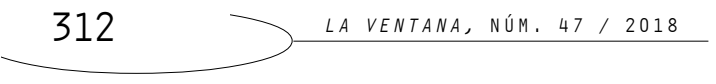

\title{
El techo de cristal en México
}

\section{María Elena Camarena Adame ${ }^{1}$ María Luisa Saavedra García}

${ }^{1}$ Universidad Nacional Autónoma de México, México. Correo electrónico: mcamarena_adame@hotmail.com ${ }^{2}$ Universidad Nacional Autónoma de México, México. Correo electrónico: maluisasaavedra@yahoo.com

Resumen

El "techo de cristal" son los obstáculos que impiden que una mujer alcance puestos de alto nivel en las organizaciones. El objetivo de este trabajo es analizar la situación actual de las mujeres en los puestos de alta dirección en el contexto mexicano, con el fin de conocer qué tanto el techo de cristal afecta su desarrollo profesional. Para ello se realizó una investigación documental recolectando información de las empresas que cotizan en la Bolsa Mexicana de Valores (BMV), así como de publicaciones recientes del sector empresarial en México. El principal hallazgo de este trabajo muestra que en promedio, las mujeres que conforman el consejo de administración en las empresas que cotizan en la BMV, sólo alcanzan el $4.56 \%$, asimismo, de las 500 mejores empresas para trabajar en México, sólo el 3\% se encuentran dirigidas por mujeres, y de las 50 mujeres más poderosas de México, sólo el 14\% trabajan como ejecutivas en una empresa. Ello evidencia que a pesar de los avances referentes a la igualdad de género, aún resulta difícil para las mujeres superar el techo de cristal.

Palabras clave: Techo de cristal, piso pegajoso, segregación vertical. 


\section{Abstract}

The "glass ceiling" is defined as the obstacles that obstruct a woman to achieve high-level positions in organizations. The aim of this paper is to analyze the current situation of women in senior management positions in the Mexican context, in order to measure how much the glass ceiling affects their professional development. For that, a documentary research was conducted by collecting information from both, companies listed on the Mexican Stock Exchange (BMV, according to its initials in Spanish) companies, as well as recent publications by the business sector in Mexico. The main finding of this study shows that on average, women who make up the Board of Directors in companies listed on the Mexican Stock Exchange companies, only reaches $4.56 \%$, additionally, of the 500 best companies to work in Mexico, only 3\% are directed by women, and, of the 50 most powerful women in Mexico, only 14\% are company executives. Which shows that despite the advances that have occurred in relation to gender equality is still difficult for women to overcome the glass ceiling.

Keywords: Glass ceiling, sticky floor, vertical segregation.

RECEPCIÓN: 12 DE ABRIL 2017/ACEPTACIÓN: 6 DE JUNIO 2017 


\section{Introducción}

Incorporar la perspectiva de género en la organización, aunque sea necesario, no resulta una tarea fácil. La complejidad del género como categoría de análisis deriva de su propia conceptualización (Barberá, Ramos, Sarrió y Candela, 2011a). La identificación del sesgo de género y su conocimiento por parte de los individuos, organizaciones y sociedad, es el primer paso para poder erradicarlo. El segundo paso es el aislamiento de sus causas y, el tercero, la voluntad de cambio (Martín, 2007, Barberá, Ramos, Sarrió y Candela, 2011b).

En este trabajo tiene como objetivo mostrar cómo el "techo de cristal" impide a las mujeres en México acceder a puestos directivos y de liderazgo. Para este fin en primer lugar se desarrolla el marco teórico, donde se aborda las temáticas: el "techo de cristal" un desafío para las mujeres; el "techo de cristal" y los puestos directivos; el "techo de cristal" en México; la mujer y el trabajo en México y el mundo; estadísticas de las mujeres empresarias en México; la mujer ejecutiva en México y la participación de la mujer en los consejos de administración de las empresas en México. En la segunda parte se presenta la metodología de la investigación, que contiene la pregunta de investigación, el objetivo y el método. En la tercera parte se presentan los resultados del análisis y recolección de datos, lo que permitirá determinar la situación de las mujeres en los puestos ejecutivos en México. Por último, se presentan las conclusiones de este trabajo. 


\section{El "techo de cristal": un desafío para las mujeres}

Dentro de las organizaciones se ha constatado la existencia del denominado "techo de cristal". Este es un término empleado desde finales de los años ochenta del siglo XX para designar una barrera invisible que impide a las mujeres altamente cualificadas, alcanzar puestos de responsabilidad en las organizaciones en las que trabajan (Morrison, White y Van Velsor, 1986; Ramos, Barberá y Sarrió, 2003). También es definido como un entramado de obstáculos invisibles que impide a las mujeres cualificadas alcanzar posiciones de poder dentro de las organizaciones (Roldán-García, Leyra Fatou y Contreras-Martínez, 2012).

En un principio, el concepto de "techo de cristal" fue utilizado para analizar la carrera laboral de mujeres que, teniendo una trayectoria profesional y un nivel de competencia alto en sus lugares de trabajo, se topaban con un freno al intentar avanzar en sus carreras (Bustos, 2002). Todo esto, como resultado de una cultura patriarcal androcéntrica ${ }^{3}$ que deri- $\quad{ }^{3}$ Centrado en el hombre. va en una discriminación de género hacia las mujeres en el ámbito laboral y les obstaculiza el acceso a cargos superiores. El denominado "techo de cristal”, según Segerman-Peck (citado en Agut y Martín, 2007), alude a una última barrera basada en prejuicios hacia las mujeres que les impide avanzar a posiciones de alto nivel. De esa manera se quedan estancadas en los niveles medios de la dirección. 
Esta barrera es la que impide a mujeres con capacidad personal y profesional alcanzar posiciones directivas y promocionarse (Ramos, Barberá y Sarrió, 2003). Las explicaciones relativas a la falta de capacidad intelectual de las mujeres hoy en día resultan obsoletas como los pilares que apuntalan el "techo de cristal". El acceso generalizado de las mujeres a los niveles educativos superiores, incluso en carreras estereotipadamente masculinas, y los buenos rendimientos obtenidos, invalidan este tipo de argumentos (Barberá, Ramos y Sarrió, 2000).

Existen diversas barreras externas, entre las que destacan los estereotipos de género, en los que persiste la creencia de que determinadas características son propias de las mujeres y hombres (Agut y Martín, 2007). A ellas las consideran como pasivas y tímidas, en oposición a los estereotipos sobre las cualidades indispensables para dirigir con éxito, asociadas a actitudes predominantemente "masculinas" (agresividad, competitividad, determinación, vigor) (Organización Internacional del Trabajo, 2014). Esto se puede observar en los puestos de trabajo que ocupan hombres y mujeres, pues ellas se siguen concentrando en determinadas profesiones ya feminizadas y en los niveles más bajos de la jerarquía. Sin embargo, se confirma que las características que a menudo se perciben como atributos "femeninos" (integridad, diligencia, sentido de la cooperación, sinceridad), se consideran cada vez más como cualidades que pueden valorizar la imagen de una organización (Organización Internacional del Trabajo, 2014a). 
Las mujeres no sólo se enfrentan a un "techo de cristal" que les impide acceder a los puestos superiores ${ }^{4}$ El suelo pegajoso, al que las mujeres se de la escala, sino también a un suelo pegajoso, ${ }^{4}$ Este sistemáticamente puestos inferiores, de concepto ilustra las dificultades que enfrentan las norsalario. mujeres graduadas para acceder a los primeros niveles de la carrera académica (Torres y Pau, 2011). Al respecto, Ramos, Barberá y Sarrió (2003), señalan factores de tipo interno y externo que estarían dificultando el ascenso de las mujeres a puestos de poder:

a) Barreras internas asociadas a la identidad de género femenina. Éstas tienen relación con el proceso de socialización que fomenta el desarrollo de características y actitudes asociadas a la identidad de género femenina que pueden ser negativas para su promoción y éxito profesional.

b) Barreras externas asociadas a la cultura organizacional y los estereotipos de género. La cultura organizacional actúa bloqueando la promoción laboral de las mujeres a través de varios procesos influidos por estereotipos y roles de género. Así tenemos: 1) las reglas informales, redes o networks masculinas existentes en las organizaciones, 2) la política de recursos humanos relativa a la selección de personal y de desarrollo de carrera de los miembros de la organización, 3) la escasez de mentorización de mujeres, y 4) la falta de políticas organizacionales tendentes a lograr un mayor respeto a 
la vida privada y a la conciliación entre el trabajo y la familia.

c) Barreras producidas por el rol reproductivo y las responsabilidades familiares. El perfil directivo imperante está asociado a largas horas de permanencia y movilidad geográfica. El triple papel de las mujeres: esposa, madre y directiva; representa un problema crucial para asumir puestos que demandan disponibilidad casi total. Por otro lado, la falta de un reparto equitativo de las responsabilidades familiares y la falta de apoyos organizacionales, impiden a las mujeres asumir puestos de poder.

Sin embargo, De Anca y Aragón (2007) encontraron que una de las barreras más importantes es la propia decisión que toma la mujer de no aceptar puestos directivos para no romper el equilibrio entre su profesión y su papel de esposa y madre.

Al respecto, un estudio elaborado en España (Cuadrado y Morales, 2007) revela que la identificación emocional con la organización, la interferencia del trabajo en la vida familiar y la identificación cognitiva con la organización y los valores propios, son las variables que tienen poder predictivo para diferenciar a las mujeres que no ocupan un puesto directivo de las que sí lo hacen. 


\section{"Techo de cristal" y los puestos directivos}

Las desigualdades de género son resultado de un reparto desigual en la distribución de responsabilidades y recursos, especialmente a través de la práctica institucional (Barberá, Ramos y Candela, 2011b). Se han establecido reglas visibles e invisibles en el entorno "masculino" al que las mujeres a veces encuentran difícil adaptarse: los colegas y los clientes no consideran automáticamente a las mujeres como iguales de los hombres; ellas generalmente deben trabajar mucho más que los hombres para probarse a sí mismas, y en ocasiones deben adaptarse más de lo necesario a los estilos masculinos de trabajo (Organización Internacional del Trabajo, 2014a).

A las mujeres se les suelen atribuir rasgos relacionados con aspectos afectivos, emocionales y tendientes a favorecer las relaciones. Por su parte, a los hombres se les atribuyen rasgos relacionados con la productividad, la eficacia, la autonomía, la independencia y la competencia para el logro de ciertas metas (Cuadrado y Morales, 2007).

Cuando las mujeres que ocupan puestos que implican mayor responsabilidad, adoptan conductas "femeninas" y otorgan importancia a los intereses comunes de los empleados y la organización, son víctimas de devaluaciones, desaprobaciones personales y sanciones sociales. Como resultado, hay casos en los que las mujeres deciden evitar ser promovidas por los costos personales que ello implica (Cuadrado y Morales, 2007). Por otra parte, cuando los puestos de poder son ocupados por una mujer, éstos se devalúan 
socialmente al no estar desempeñados por el sexo masculino. Sin embargo, cuando las mujeres ejercen actividades de poder son sancionadas al ser consideradas masculinas. Aunque son los individuos los que "construyen el género" debido a factores culturales y sociales (Barberá, Ramos y Candela, 2011a).

${ }^{5}$ La segregación horizontal se refiere a la escasa participación de la mujer en determinados sectores económicos, áreas o departamentos. Se produce al través del reclutamiento. La segregación vertical. Se refiere a la menor presencia de mujeres en los puestos directivos o de responsabilidad (Forética, 2011, citado en Camarena y Saavedra, 2016).

La segregación vertical y horizontal ${ }^{5}$ producida por estereotipos sobre la mujer en su vida profesional perjudica de modo considerable su proyección en este ámbito. Los estereotipos masculinos de agresividad y competitividad siguen siendo las cualidades más demandadas para el liderazgo. Parece que los inhibidores fundamentales a la hora de la ascensión de la mujer a puestos directivos pueden ser barreras externas, bien sea sociales (estereotipos y falta de modelos femeninos), o barreras organizacionales (falta de flexibilidad en la empresa y por tanto dificultad para la conciliación) (De Anca y Aragón, 2007).

Por lo anterior, son pocas las mujeres que ascienden a los puestos clave de poder después de haber vencido numerosas dificultades. Las que llegan, habitualmente, se ven presionadas a asumir un modelo de dirección masculino. Sin embargo, pese a que el acceso a estos puestos es complicado, no se puede calificar de imposible en tanto las mujeres dispongan de recursos que les ayuden a afrontar estos obstáculos, reduciéndolos o eliminándolos (Agut y Martín, 2007). 


\section{El "techo de cristal" en México}

De acuerdo con Barragán, Mills y Runte (2011) los aspectos históricos y culturales de la formación de la sociedad mexicana tienen implicaciones en cómo se perciben a sí mismas las personas y cómo perciben la igualdad de oportunidades. Así pues, la familia es una institución muy importante en la sociedad. Después de la conquista, México se convirtió en un país católico en el que los valores dominantes son la familia y las tradiciones. A partir de entonces aparecen el machismo y el marianismo para marcar los roles de género. En primer lugar el machismo es una forma exagerada de masculinidad y responsabilidad por la familia, pero sobre todo de dominio. En segundo lugar el marianismo es un modelo ideal para la condición de la mujer, presenta la imagen de la sumisa y santa mujer dedicada a su familia como un ideal que otras mujeres deben emular.

Los roles de género en la cultura mexicana han tenido una evolución histórica, especialmente en términos de los derechos de las mujeres a la igualdad de oportunidades. Tras la Revolución de 1910 a las mujeres no se les permitía votar por miedo a su alianza con la Iglesia Católica, que fue visto como un enemigo de los ideales de la revolución. Fue hasta 1953 en que adquirieron su derecho al voto; sin embargo, por muchos años fueron excluidas de ocupar cargos públicos y de realizar trabajos que eran tradicionalmente masculinos. Finalmente en 1974 una enmienda a la constitución instituyó la igualdad de oportunidades para hombres y mujeres (Barragán, Mills y Runte, 2011). 
La globalización, el Tratado de Libre Comercio y el ingreso de empresas transnacionales al país han creado nuevos discursos sobre derechos humanos e igualdad de oportunidades fomentando la equidad. Sin embargo, de acuerdo con el World Economic Forum (2014), la brecha de género en México aún persiste y se agudiza en el aspecto económico. De acuerdo con el texto anteriormente citado el país quedó en el lugar 120 de 142.

\section{La mujer y el trabajo en México y el mundo}

A pesar de que la mujer ha aumentado su participación en el mercado de trabajo en las últimas décadas, su posición es de desventaja con respecto a sus compañeros. Esta desigualdad se ve reflejada en su dificultad para acceder a puestos de poder debido al fenómeno del "techo de cristal", basado en prejuicios hacia las mujeres que les dificulta y/o impide llegar a la cima (Agut y Martín, 2007). Las mujeres deben hacer frente a un mayor número de barreras que los hombres en el mercado de trabajo, especialmente cuando se trata del acceso a puestos de mayor responsabilidad (Cuadrado y Morales, 2007). A pesar de que la participación de las mujeres en el mercado laboral generalmente fomenta la honestidad, esto es debido a cuestiones históricas ya que culturalmente al hombre le cuesta más trabajo intentar sobornar o corromper a una mujer (Centro de Investigación de la Mujer en la Alta Dirección, 2013).

La situación global de las mujeres en materia de empleo no ha evolucionado significativamente desde el 2001. Las tendencias 
mundiales del empleo indican que las mujeres continuaban teniendo menores índices de participación en el mercado de trabajo, mayores tasas de desempleo y significativas diferencias de remuneraciones, en comparación con los hombres (Organización Internacional del Trabajo, 2014b). Para el año 2012, la proporción de mujeres en empleo vulnerable (trabajadores familiares no remunerados y trabajadores por cuenta propia) era de $50 \%$ y la de los hombres de $48 \%$. En cuanto a la brecha de género en el desempleo esta fue de 0.7 puntos porcentuales para el año 2012. Es decir, la destrucción de 13 millones de puestos de trabajo para las mujeres (Organización Internacional del Trabajo, 2012).

La idea de que las mujeres son menos ambiciosas que los varones o que no tienen madera para dirigir favorece, por un lado, el hecho de que las organizaciones las valoren menos y, se resistan a contratarlas. Por otro lado incide en que ellas lleguen a creerse que no sirven, con lo cual pueden esforzarse menos y poner menos empeño en su promoción profesional (Barberá, Ramos, Sarrió y Candela, 2011a). No obstante la mayor facilidad de las mujeres para mostrar emociones, sentimientos y empatía, constituyen recursos humanos que pueden ser benéficos tanto para el clima de la organización como para la mayor efectividad del trabajo en equipo.

En México, la Encuesta Nacional de Empleo 2014 reportó que por cada 100 hombres mayores de 14 años, 77 son económicamente activos; mientras que, de cada 100 mujeres en las mismas condiciones, sólo 42 forman parte de la población económicamente activa (INEGI, 2014). 
Las mujeres mexicanas se enfrentan a importantes obstáculos que les impiden participar totalmente en el mercado laboral y sobre todo en puestos de decisión. Algunos de estos obstáculos son la carga del trabajo no remunerado, los tradicionales roles de género y la carencia de políticas de conciliación entre trabajo y vida familiar, especialmente la insuficiente oferta de servicios de cuidado infantil y de prácticas laborales flexibles (Centro de Investigación de la Mujer en la Alta Dirección, 2013; De Anca y Aragón, 2007).

\section{Mujeres empresarias en México}

En México de cada cinco pequeñas y medianas empresas que se abren, tres están lideradas por mujeres (Centro de Investigación de la Mujer en la Alta Dirección, 2013) y el 37\% del Producto Interno Bruto en México es aportado por mujeres (INEGI, 2013). En 2008 el diagnóstico de Inmujeres reportó un incremento de participación de empresarias en el sector de los servicios (48\%) y un porcentaje relativamente menor en el sector de comercio e industria ( $21 \%$ y $15 \%$, respectivamente). Como puede observarse, todavía predomina el hecho de que las empresarias participan en actividades consideradas una extensión de las tareas domésticas. Sin embargo, las emprendedoras encuentran menos problemas que los emprendedores a la hora de encontrar el personal adecuado, localizar materias primas o dar con la tecnología necesaria para la puesta en marcha de su negocio. Los empresarios señalan la disponibilidad de fondos como un motivador para la puesta en marcha 


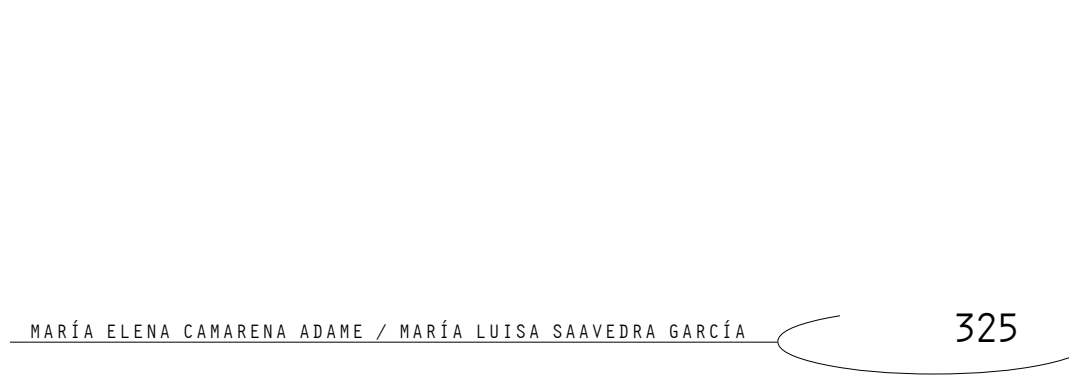

de su empresa con mayor intensidad que las empresarias (Belso, 2005). Por otra parte, las empresas dirigidas por mujeres encuentran obstáculos desproporcionados al momento de enfrentarse a la realidad empresarial como: tasas de interés más elevadas al momento de solicitar un crédito al no contar con un patrimonio propio que las respalde, acceso limitado a redes empresariales por no contar con experiencia laboral previa (Camarena y Saavedra, 2016).

En 2014, poco más de 2 millones de las personas insertas en el mercado de trabajo correspondía a empleadores/as; es decir, sólo 4.3\% del total de la población ocupada en el mercado laboral (5.6\% del total de la población masculina y $2.4 \%$ del total de la femenina). Para mediados de 2014, $86.3 \%$ de las mujeres empleadoras y $72.8 \%$ de los hombres con esta característica están al frente de micronegocios, lo que muestra el predominio de estas unidades económicas, principalmente entre las mujeres (INEGI, 2014; Instituto Nacional de las Mujeres, 2014).

En lo que se refiere al nivel de escolaridad, las estadísticas reportan que $36.5 \%$ de las mujeres empresarias tienen nivel medio superior o superior y $34 \%$ de las mujeres empresarias tienen un nivel de escolaridad de secundaria completa. Por otro lado el $70 \%$ inicia su empresa con ahorros personales, $88 \%$ de las empresas son administradas sin ayuda profesional, $72 \%$ tiene como cliente al consumidor final, y más del 50\% no realiza publicidad (Tarango, 2013; INEGI, 2013; Instituto Nacional de las Mujeres, 2014).

El nivel de ingresos de las mujeres empleadoras es menor que el de los hombres empleadores. Así pues, mientras que 12.5\% de las 
mujeres empleadoras obtienen ingresos de hasta un salario mínimo, $8.6 \%$ de los hombres tiene este nivel Contrariamente la proporción de hombres empleadores que ganan más de 5 salarios mínimos asciende a $25 \%$, mientras que en mujeres empleadoras llega a $17.4 \%$ (Instituto Nacional de las Mujeres, 2014). Es importante señalar que la desigualdad de salarios es más grande entre los que ocupan puestos de funcionarios públicos y gerentes del sector privado, donde la igualdad de salarios se lograría incrementando el sueldo de la mujer en un 25.6\% (Camarena, Saavedra y Ducloux, 2015).

\section{La mujer ejecutiva en México}

Las mujeres ocupan el 31\% de los puestos de alta dirección en México (32\% en la OCDE), 7\% de los miembros de la junta directiva de las empresas mexicanas son mujeres (10\% en la OCDE), y sólo el 2\% de las mujeres mexicanas son empresarias (en comparación con el 6\% de los hombres) (Gurría, 2013). Ellas ocupan menos de un tercio de los puestos gerenciales y tienen una participación de 1 mujer por cada 10 hombres en los puestos directivos. (Organización Internacional del Trabajo, 2014a).

La Asociación Mexicana en Dirección de Recursos Humanos (AMEDIRH), señala que en los últimos 15 años la participación de las mujeres en puestos de dirección en las principales empresas del país avanzó de 43\% a 52\%. Esto significa que el papel de la mujer en el mundo laboral es crucial para el país, ya que representa una contribución fundamental en el cumplimiento de los objetivos de 
desarrollo social, educativo, comunitario y económico (Tarango, 2013).

Si se considera a las mujeres en los principales cargos ejecutivos de las grandes empresas en México puede verse que su presencia se concentra, en primer lugar, en ciertas áreas como la dirección de recursos humanos y compras. En segundo y tercer lugar, la presencia de las mujeres ejecutivas también es importante en las direcciones de comunicación y relaciones públicas, y en actividades relacionadas con ventas y finanzas (Zabludovsky, 1997). Al contrario, hay pocas posibilidades de encontrar mujeres en los puestos de decisión relacionados con la producción. Otras actividades donde la mujer tiene escasa participación son en el ámbito jurídico, medio ambiente y control de calidad.

Se ha demostrado cómo a medida que se asciende en la pirámide organizacional, la presencia de la mujer disminuye (Hernández, 2010). Esta brecha en los cargos de alta dirección se explica tanto por factores culturales compartidos en toda la sociedad, como por elementos específicos relacionados con la cultura organizacional (Zabludovsky, 1997). Según Martínez (2004) la escasa presencia de mujeres en la dirección de las organizaciones empresariales obedece a dos cuestiones fundamentales:

- 1) La presencia de empresas dirigidas por mujeres no tiene el peso económico suficiente para que la élite directiva las considere como candidatas potenciales a un puesto de dirección. 
2) Las mujeres empresarias no participan activamente, ni muestran interés por hacer política al interior de las cámaras patronales.

Pese a que los factores que tradicionalmente han limitado el desarrollo profesional de la mujer, como el acceso a la formación universitaria, apenas es un obstáculo, la menor presencia de la mujer en los puestos de responsabilidad es cada vez más un problema de decisiones, no de opciones. Así, las mujeres consideran que los principales frenos que limitan su carrera profesional son los personales y que las principales causas de la escasa presencia femenina en posiciones de alta dirección son también personales (De Anca y Aragón, 2007). La dificultad para conciliar el trabajo directivo con las responsabilidades familiares aparece como un obstáculo clave en el ascenso y promoción de las mujeres. La conclusión más generalizada es que cualquier acción para incrementar la presencia de mujeres en puestos directivos exige, como condición necesaria para ser efectiva, aligerar las responsabilidades familiares y compartir con los varones las cargas domésticas (Barberá, Ramos y Sarrió, 2000; Kauffman, 2009).

Un aspecto importante a considerar es que las mujeres ejecutivas tienen mayor habilidad para mantener relaciones personales más estrechas con los demás, tienen en cuenta tanto los sentimientos como los datos básicos al momento de tomar decisiones y buscan soluciones en las que todos ganen evitando situaciones en las que alguien tenga que perder. También subordinan los progresos 
personales de corto plazo a la mejora de la organización a largo plazo (Ramos, Barberá y Sarrió, 2003).

\section{La participación de la Mujer en los Consejos de}

Administración de las empresas en México

La diversidad de género en el Consejo de Administración (CA) de las empresas podría mejorar la calidad de los debates e incrementar la capacidad de éste en la supervisión del proceso de divulgación de información corporativa. La diversidad de género en el CA está asociada a una mayor calidad de las deliberaciones y a una comunicación más efectiva (McInerney-Lacombe, Bilimoria y Salipante, citados por Briano y Saavedra, 2015), lo cual incide en un incremento en la diseminación de información hacia los accionistas. También, la participación femenina en el cA genera un mayor control sobre las acciones gerenciales y la transparencia corporativa, a través de una mayor asistencia en las reuniones del CA y en los comités de auditoría así como el incremento de las prácticas de buen gobierno adoptadas (Hillman, Shropshire y Cannella, citados por Briano y Saavedra, 2015).

$\mathrm{Al}$ respecto de la participación de las mujeres en el CA de las empresas se encontró que en México de una muestra de 50 empresas que cotizan en la Bolsa Mexicana de Valores, sólo el 7\% de los miembros titulares son mujeres y este porcentaje aumenta $12 \%$ cuando los miembros son suplentes (Hernández, Camarena y Castanedo, 2009). 


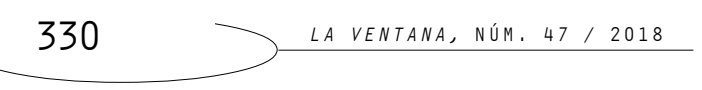

\section{Metodología de la Investigación}

\section{Pregunta de investigación}

¿Cuál es la situación actual de las mujeres en los puestos de alta dirección en el contexto mexicano?

\section{Método}

Se realizó una investigación documental, para lo cual se analizó la base de datos de las empresas que cotizan en la BMV, con el fin de determinar la conformación de su consejo directivo. También se revisaron y utilizaron las bases de datos de dos importantes publicaciones: 1) Las 500 mejores empresas para trabajar en México y, 2) Las 50 mujeres más poderosas de México.

\section{La situación de las mujeres en puestos ejecutivos en México}

\section{Análisis de las 500 mejores empresas para trabajar}

De las 500 mejores empresas para trabajar en México (Graglia, 2014) solo 16 se encuentran dirigidas por mujeres. Esto representa el 3.2\%, como se muestra en el cuadro siguiente: 
Cuadro 1. Empresas dirigidas por mujeres, de las 500 mejores empresas más importantes de México

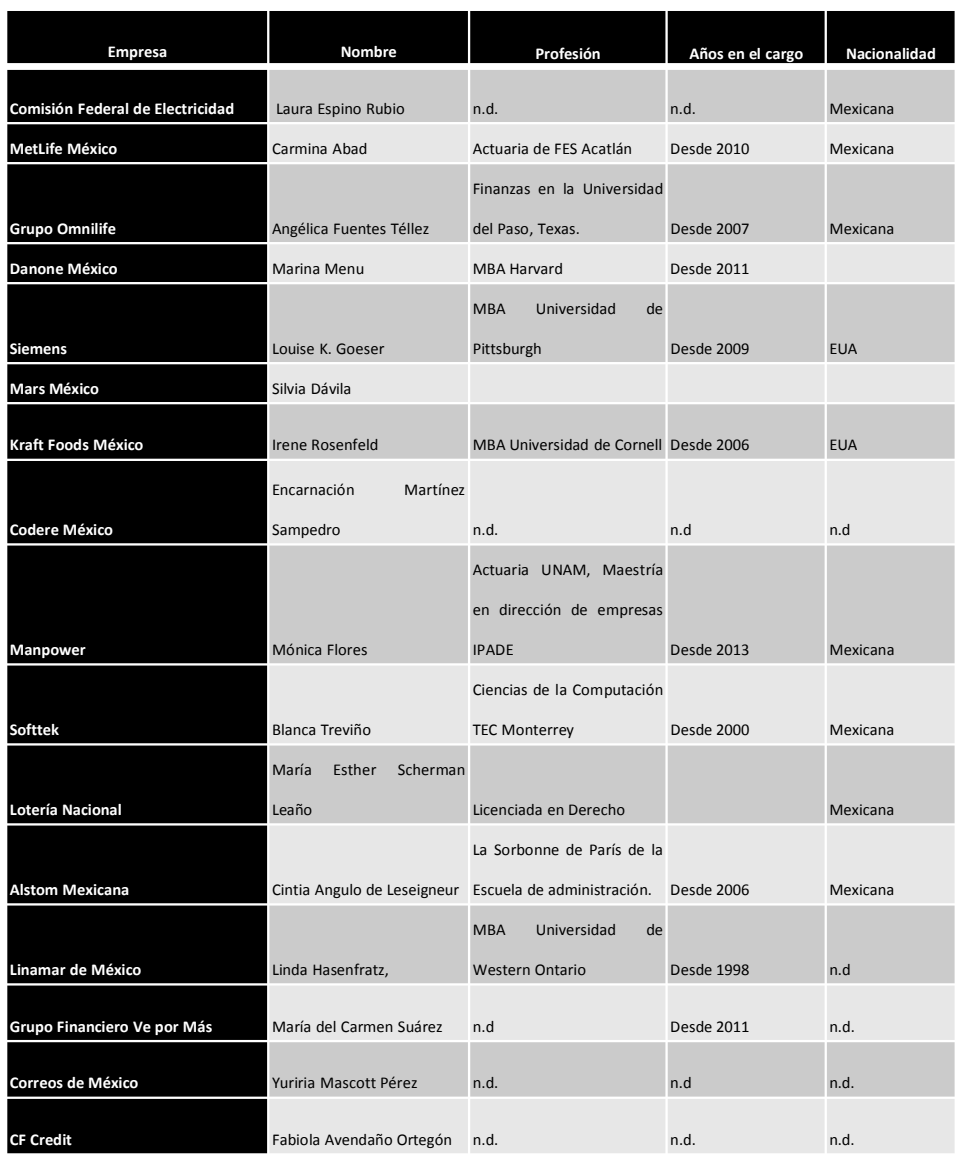

Fuente: Elaboración propia a partir de Gralia (2014).

n.d.: Dato no disponible. 
Como puede observarse en el Cuadro 1, todas las mujeres que son líderes empresariales cuentan con estudios superiores, una buena parte de ellas (seis) en universidades extranjeras. A su vez resulta necesario puntualizar algunos aspectos:

- Paula Santilli estuvo de 2009 a 2011 en Pepsico Bebidas actualmente se encuentra en Botanas Sabritas (Esta no fue seleccionada en 2014, dentro de las 500 empresas más importantes de México).

Grace Lieblein, de General Motors, estuvo muchos años en la misma empresa, desde estudiante. Ahora es vicepresidenta de Calidad Global en General Motors.

- Nicole Reich de Polignac, de Scotiabank, presentó su renuncia después de 5 años en el puesto. Esta fue otorgada de inmediato, contrario a lo que marcan los protocolos bancarios en donde dice que pasa un plazo de 6 meses a un año y medio desde que los directores generales presentan su renuncia hasta que dejan definitivamente el puesto.

-En Omnilife, Angélica Fuentes es esposa del dueño de esta empresa. Antes de ocupar esa posición fue directora general del Grupo Imperial, considerada la compañía de gas más importante en el norte de México y de la cual todavía es accionista y miembro del consejo directivo. 
Ninfa Salinas, directora general de Energía y Sustentabilidad del Grupo Salinas, es hija de Ricardo Salinas Pliego.

En Kraft, su directora a nivel internacional es mujer, pero en México no.

Es necesario hacer notar que cuando una mujer es removida de su puesto de directora general, la mayoría de las veces no lo anuncian a los medios de comunicación ni presentan informes, como sucede en caso de los hombres. Sólo dicen quién es el nuevo (fue el caso de PepsiCo). Generalmente, las mujeres son removidas en casos de dificultades en la empresa.

\section{Análisis de las 50 mujeres más poderosas de México}

De las 50 mujeres más poderosas de México en 2014 (Forbes, 2014), sólo 17 son empresarias o ejecutivas, como se muestra en el cuadro siguiente: 
Cuadro 2. Mujeres ejecutivas dentro de las 50 mujeres más poderosas de México

\begin{tabular}{|c|c|c|}
\hline Ranking & Nombre & Puesto \\
\hline 1 & $\begin{array}{l}\text { María } \\
\text { Asunción } \\
\text { Aramburuzabal } \\
\text { a }\end{array}$ & $\begin{array}{l}\text { Empresaria } \\
\text { inversionista/CEO Tresalia }\end{array}$ \\
\hline 2 & \begin{tabular}{ll|} 
Eva & Gonda \\
Rivera &
\end{tabular} & Empresaria/FEMSA \\
\hline 3 & Blanca Treviño & $\begin{array}{l}\text { Empresaria/presidenta y CEO } \\
\text { Sofftek }\end{array}$ \\
\hline 4 & $\begin{array}{l}\text { Angélica } \\
\text { Fuentes Téllez }\end{array}$ & $\begin{array}{l}\text { Empresaria/presidenta } \\
\text { Grupo Omnilife }\end{array}$ \\
\hline 5 & $\begin{array}{l}\text { Gabriela } \\
\text { Hernández }\end{array}$ & $\begin{array}{l}\text { Empresaria/presidenta } \\
\text { Grupo Omnilife }\end{array}$ \\
\hline 8 & Salma Hayek & Actriz y empresaria \\
\hline 7 & $\begin{array}{l}\text { Martha } \\
\text { Debayle }\end{array}$ & Locutora y empresaria \\
\hline 8 & $\begin{array}{l}\text { Cintia Angulo } \\
\text { Leseigneur }\end{array}$ & $\begin{array}{l}\text { Presidenta y directora } \\
\text { general de Alstom Mexicana }\end{array}$ \\
\hline 9 & $\begin{array}{l}\text { Alejandra } \\
\text { Palacios }\end{array}$ & $\begin{array}{l}\text { Comisionada de presidente } \\
\text { de COFECE }\end{array}$ \\
\hline 10 & $\begin{array}{l}\text { Adriana } \\
\text { Labardini }\end{array}$ & Comisionada del IFT \\
\hline 11 & $\begin{array}{l}\text { Luz Adriana } \\
\text { Ramírez } \\
\text { Chávez }\end{array}$ & $\begin{array}{l}\text { Directora general de Visa } \\
\text { México }\end{array}$ \\
\hline 12 & $\begin{array}{l}\text { Gabriela } \\
\text { Warkentin }\end{array}$ & $\begin{array}{l}\text { Académica, directora de } \mathrm{W} \\
\text { Radio }\end{array}$ \\
\hline 13 & Lorena Ochoa & Empresaria \\
\hline 14 & $\begin{array}{l}\text { Martha } \\
\text { Higareda }\end{array}$ & Actriz, empresaria \\
\hline 15 & Carmina Abad & Directora General Metlife \\
\hline
\end{tabular}




\begin{tabular}{|c|c|c|}
\hline 16 & $\begin{array}{l}\text { Marinela Servitje de } \\
\text { Lerdo de Tejada }\end{array}$ & $\begin{array}{l}\text { Empresaria promotora } \\
\text { cultural }\end{array}$ \\
\hline 17 & $\begin{array}{ll}\text { Rosario } & \text { Robles } \\
\text { Berlanga } & \end{array}$ & Titular de SEDESOL \\
\hline 18 & Zélika García & $\begin{array}{l}\text { Directora general de } \\
\text { Zona Maco }\end{array}$ \\
\hline 19 & Claudia Ruiz Massieu & Secretaria de Turismo \\
\hline 20 & Alejandra Lagunes & $\begin{array}{ll}\text { Coordinadora } & \text { de la } \\
\text { Estrategia } & \text { Nacional } \\
\text { Digital } & \\
\end{array}$ \\
\hline 21 & $\begin{array}{ll}\text { Mónica } & \text { Flores } \\
\text { Barragán } & \end{array}$ & $\begin{array}{l}\text { Directora general de } \\
\text { Manpowergroup Latam }\end{array}$ \\
\hline 22 & $\begin{array}{l}\text { Sandra Sánchez y } \\
\text { Oldenhage }\end{array}$ & $\begin{array}{l}\text { Directora general } \\
\text { adjunta de Probiomed }\end{array}$ \\
\hline 23 & $\begin{array}{l}\text { Patricia } \quad \text { Mercado } \\
\text { Castro }\end{array}$ & $\begin{array}{l}\text { Secretaria de Trabajo en } \\
\text { el GDF }\end{array}$ \\
\hline 24 & $\begin{array}{l}\text { Ximena Puente de la } \\
\text { Mora }\end{array}$ & $\begin{array}{l}\text { Consejera presidente del } \\
\text { IFAI }\end{array}$ \\
\hline 25 & Alondra de la Parra & Directora de orquesta \\
\hline 26 & Nina Zambrano & $\begin{array}{llr}\text { Directora general del } & \text { de } \\
\text { Museo de } & \text { Arte } \\
\text { Contemporáneo } & \text { de } \\
\text { Monterrey } & \end{array}$ \\
\hline 27 & $\begin{array}{l}\text { Ivonne } \\
\text { Ortega }\end{array}$ & $\begin{array}{l}\text { Secretaria general del } \\
\text { CEN del PRI }\end{array}$ \\
\hline
\end{tabular}

Fuente: Elaboración propia con base en: Forbes (2014).

De las 50 mujeres más poderosas de México 27 son ejecutivas o empresarias, específicamente 10 son empresarias, 7 son ejecutivas de alto nivel en empresas privadas (14\%) y 10 ocupan altos cargos en instituciones públicas. Esto se debe al efecto del "techo de cristal" 
donde según lo señalan Chávez, N. y Ríos (2014) Los autores encontraron evidencias de una clara preferencia por parte de los empleadores por contratar más hombres que mujeres en los cargos jerárquicos, e incluso la preferencia por mujeres solteras y sin hijos. Estos hallazgos son coincidentes con lo encontrado en España por Castaño, Martín, Vázquez, y Martínez (2010), respecto a que las mujeres siguen estando poco representadas en los puestos de alto nivel. Según los investigadores las principales dificultades a las que se enfrentan son: no tienen mentores o modelos a seguir, porque las redes de mentores en las organizaciones son predominantemente masculinas; tienen menos experiencia y antigüedad en el trabajo; y se encuentran limitadas para dedicar tiempo al trabajo fuera de casa en viajes de negocios.

\section{Análisis del Consejo de Administración de las Empresas que cotizan en la Bolsa Mexicana de Valores (BMV)}

Se analizaron 107 empresas que cotizan en la BMV, en cuanto a su conformación de hombres y mujeres que forman parte del Consejo de Administración. Del total de las 107 empresas analizadas se obtuvo que la suma de los integrantes del CA son: 1426 personas, de las cuales 1361 son hombres y 65 son mujeres. Esto indica que solo el $4.56 \%$ de los consejeros de estas empresas son mujeres. Por su parte, de las 107 empresas analizadas, solo 45 cuentan con mujeres participando en su CA. El análisis de estos datos se presenta en el Cuadro 3, donde se considera sólo a las empresas que cuentan con mujeres en el CA. 
Cuadro 3. Mujeres en el Consejo de Administración de empresas que cotizan en la BMv

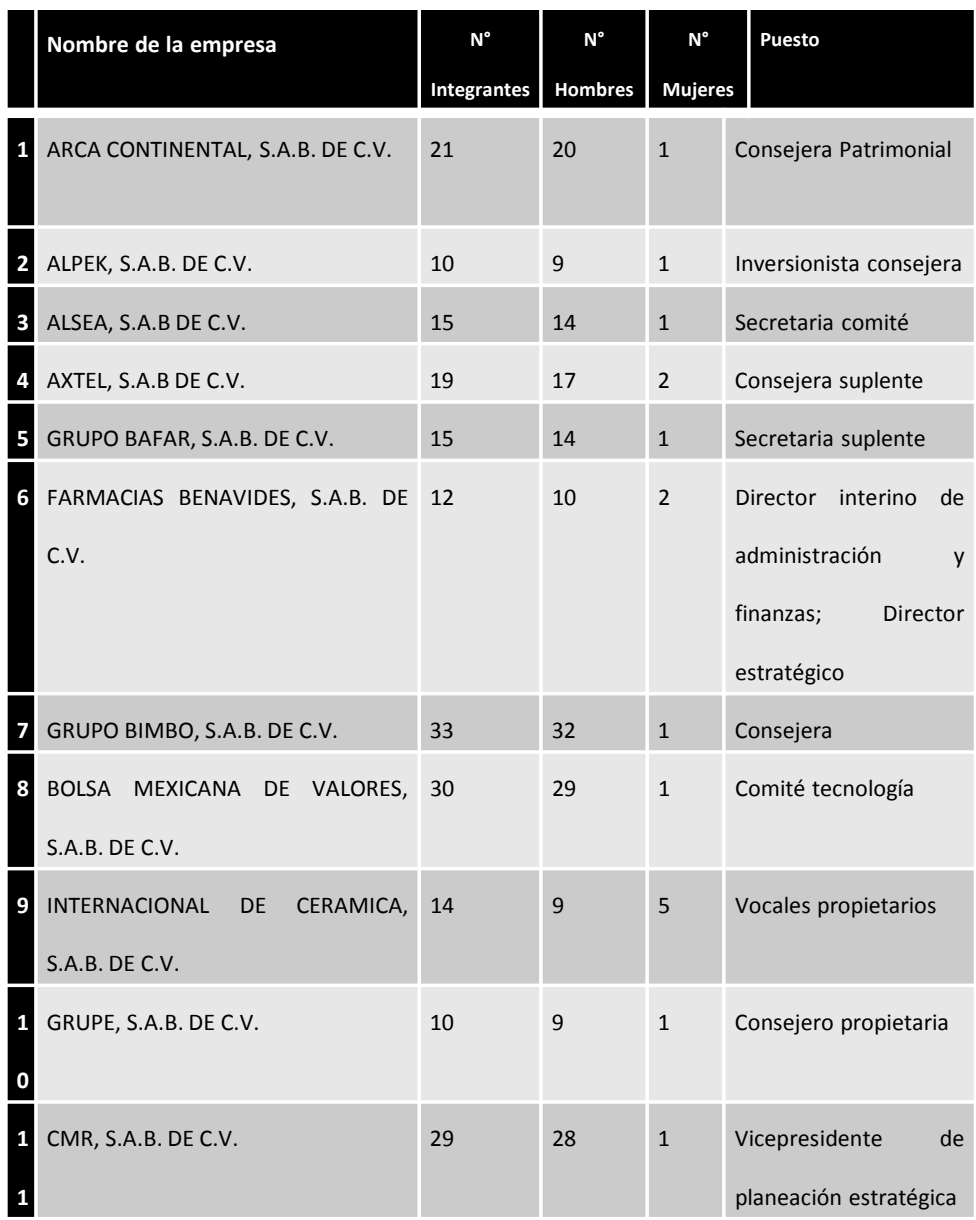




\begin{tabular}{|c|c|c|c|c|c|}
\hline 1 & $\begin{array}{l}\text { CREDITO REAL, S.A.B DE C.V., SOFOM, } \\
\text { E.R. }\end{array}$ & 18 & 17 & 1 & Consejera suplente \\
\hline & ORGANIZACIÓN CULTIBA, S.A.B. DE C.V. & 19 & 17 & 2 & Consejero \\
\hline 1 & CYDSA, S.A.B DE C.V. & 17 & 15 & 2 & $\begin{array}{l}\text { Consejero; delegada del } \\
\text { consejo }\end{array}$ \\
\hline 1 & $\begin{array}{l}\text { FOMENTO ECONÓMICO MEXICANO, } \\
\text { S.A.B.DE C.V. }\end{array}$ & 17 & 14 & 3 & Inversionista privada \\
\hline 6 & $\begin{array}{l}\text { FINANCIERA INDEPENDENCIA, S.A.B. DE } \\
\text { C.V., SOFOM, E.N.R. }\end{array}$ & 14 & 12 & 2 & $\begin{array}{l}\text { Consejeras propietarias, } \\
\text { hijas del presidente del } \\
\text { consejo }\end{array}$ \\
\hline 1 & CORPORATIVO FRAGUA, S.A.B. DE C.V. & 10 & 7 & 3 & $\begin{array}{l}\text { Vicepresidenta; } \\
\text { consejera propietaria y } \\
\text { secretaria }\end{array}$ \\
\hline 1 & FRESNILLO PLC & 12 & 11 & 1 & Consejera independiente \\
\hline 1 & CORPORATIVO GBM, S.A.B. DE C.V. & 23 & 22 & 1 & Prosecretaria \\
\hline 2 & GENTERA, S.A.B. DE C.V. & 13 & 12 & 1 & Consejera propietaria \\
\hline 2 & $\begin{array}{l}\text { GRUPO FINANCIERO INBURSA, S.A.B. } \\
\text { DE C.V. }\end{array}$ & 18 & 17 & 1 & $\begin{array}{l}\text { Consejero propietaria } \\
\text { independiente }\end{array}$ \\
\hline
\end{tabular}




\begin{tabular}{|c|c|c|c|c|c|}
\hline 2 & $\begin{array}{l}\text { GRUPO FINANCIERO } \\
\text { INTERACCIONES, S.A. DE C.V. }\end{array}$ & 12 & 11 & 1 & $\begin{array}{l}\text { Consejera Patrimonial, } \\
\text { hija del presidente del } \\
\text { consejo }\end{array}$ \\
\hline 3 & $\begin{array}{l}\text { GRUPO FINANCIERO BANORTE, S.A.B. } \\
\text { DE C.V. }\end{array}$ & 16 & 14 & 2 & $\begin{array}{l}\text { Consejera propietaria } \\
\text { patrimonial; Consejera } \\
\text { patrimonial } \\
\text { independiente }\end{array}$ \\
\hline 2 & GRUPO MODELO, S.A.B. DE C.V. & 11 & 10 & 1 & Consejero propietaria \\
\hline 2 & GRUPO HERDEZ, S.A.B. DE C.V. & 10 & 9 & 1 & Consejera Patrimonial \\
\hline 2 & EMPRESAS ICA, S.A.B DE C.V. & 11 & 9 & 2 & $\begin{array}{l}\text { Consejera } \\
\text { independiente }\end{array}$ \\
\hline 2 & COCA-COLA FEMSA, S.A.B. DE C.V. & 11 & 10 & 1 & Inversor privado \\
\hline 2 & $\begin{array}{l}\text { GENOMMA LAB INTERNACIONAL, } \\
\text { S.A.B DE C.V. }\end{array}$ & 10 & 9 & 1 & Vicepresidenta \\
\hline 9 & GRUPO LAMOSA, S.A.B. DE C.V. & 14 & 13 & 1 & $\begin{array}{l}\text { Directora de María } \\
\text { Elosúa Diseño }\end{array}$ \\
\hline $\mathbf{0}$ & $\begin{array}{l}\text { EL PUERTO DE LIVERPOL, S.A.B. DE } \\
\text { C.V. }\end{array}$ & 21 & 20 & 1 & Vicepresidente \\
\hline 3 & $\begin{array}{l}\text { GRUPO AEROPORTUARIO DEL } \\
\text { CENTRO NORTE, S.A.B. DE C.V. }\end{array}$ & 11 & 10 & 1 & $\begin{array}{l}\text { Consejera } \\
\text { independiente }\end{array}$ \\
\hline
\end{tabular}




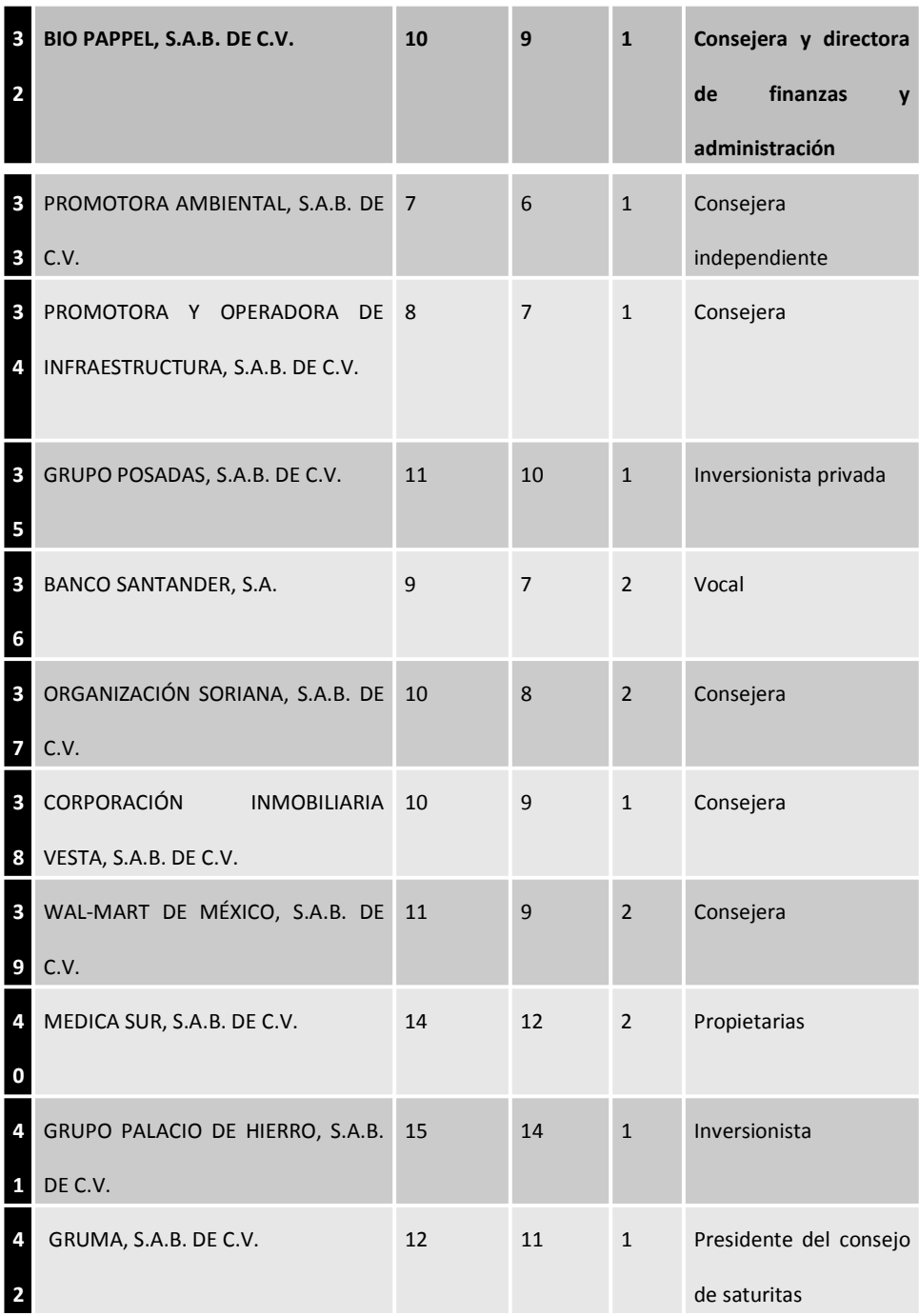



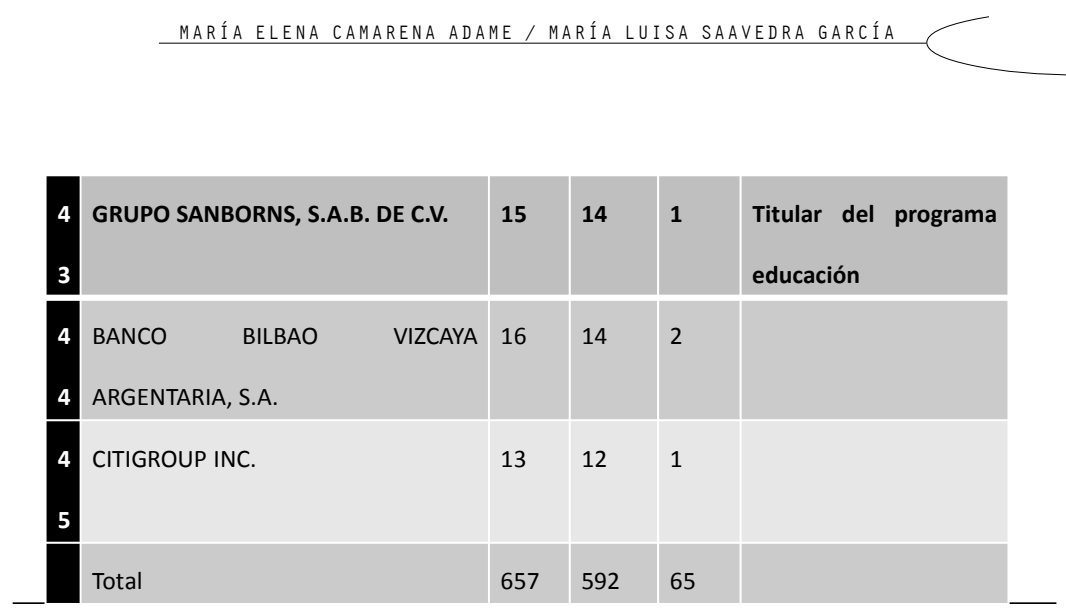

Fuente: Elaboración propia con datos de la BMV.

En el Cuadro 3 se observa que en la Financiera Independencia existen dos consejeras propietarias que son hijas del presidente del Consejo. En el Corporativo Fragua participan tres mujeres ocupando los puestos de vicepresidenta, consejera propietaria y secretaria, la empresa está integrada por familia. Por su parte el Grupo Financiero Interacciones cuenta con una mujer como consejera patrimonial, quien es la hija del presidente del consejo; y en el caso de la empresa GENOMMA LAB, participa como miembro del consejo la vicepresidenta, quien es la hermana del presidente. La descripción de estas empresas se obtuvo de los informes anuales de los accionistas, quizá existan más casos como estos que no se encuentran señalados en los informes anuales. Teles hallazgos son consistentes con la amplia brecha de género en los CA de Estados Unidos que encontraron Adams y Funk (2012).

Por otro lado, estos resultados no son coincidentes con los hallazgos de Hernández, Camarena y Castanedo (2009), quienes 
encontraron un porcentaje mayor de mujeres participando en el CA de las empresas mexicanas (7\%) que cotizan en la BMV. Esto podría deberse a que estos autores utilizaron una muestra más pequeña de 50 empresas.

\section{Conclusiones}

El "techo de cristal" es todavía un desafío muy grande para las mujeres en México dado que aún persiste la cultura patriarcal androcéntrica, la cual deriva en una discriminación de género. Esto incide en que a muchas mujeres se les obstaculice el acceso a cargos superiores. En la actualidad ya no resultan válidos los pilares que apuntalaban el "techo de cristal". Como la falta de capacidad intelectual de las mujeres, los estereotipos de género donde persiste la creencia de que determinadas características son propias de las mujeres y hombres, las dificultades que encuentran las mujeres graduadas para acceder a los primeros lugares de la carrera académica. La inequidad de género en los espacios de poder es el resultado del reparto desigual en la distribución de responsabilidades y recursos.

En este trabajo se encontró que de las 500 mejores empresas de México, solo 16 están lideradas por mujeres y cuentan con estudios superiores, lo cual demuestra que los hombres siguen ocupando la mayor parte de los puestos directivos y son altos ejecutivos, mientras que las mujeres siguen relegadas a puestos inferiores.

Así también, de las 50 mujeres más poderosas de México, 10 son empresarias, 7 son ejecutivas de alto nivel en empresas privadas y 10 
ocupan altos cargos en instituciones públicas. Es decir, las mujeres que han sido exitosas como ejecutivas de alto nivel en este grupo de 50 , solo representan $14 \%$.

Los resultados muestran que la participación de las mujeres en el CA de las empresas que cotizan en la BMV es muy baja todavía, (4.56\%). Esto implica que las empresas estarían perdiendo los beneficios de contar con mujeres en su CA y con ello contar con una mejor comunicación, mejor control y mejores prácticas de gobierno corporativo, los cuales benefician principalmente a los accionistas minoritarios de estas empresas, como lo señalan Briano y Saavedra (2015).

\section{Bibliografía}

Adams, R. y Funk, P. (2012). Beyond the Glass Ceiling: Does Gender Matter? Management Science 58(2):219-235, doi: dx.doi.org/ 10.1287/ mnsc. 1110. 1452.

Agut, S. y MarTín, P. (2007). Factores que dificultan el acceso de las mujeres a puestos de responsabilidad: una revisión teórica. Apuntes de psicología., 25 (2), 201-214.

Barberá, E.; Ramos, A., Sarrió, M. y Candela, C. (2011a). Laberinto de cristal en el liderazgo de las mujeres. Psicothema, 23(2), 173-179.

Barberá, E., Ramos, A., Sarrió, M. y Candela, C. (2011b). Más allá del "techo de cristal" Diversidad de género. Revista del ministerio de trabajo y asuntos sociales, España (40), 55-68.

— Ramos, A. y Sarrio, M. (2000). Mujeres directivas antes el tercer milenia: el proyecto NowDI XXI. Papeles del Psicólogo (75), 46-52. 
Barragán, S.; Mills, A. y Runte, M. (2011). The mexican glass ceiling and the Construction of equal opportunities: Narratives of women managers. Journal Workplace Rights, 15(3-4) 255-277.

Belso, J. (2005). Discriminación de género y fomento de nuevas empresas: Conclusiones a partir de un análisis multivariante sobre las pymes valencianas de reciente creación. Revista del ministerio de trabajo y asuntos sociales (41), 15-38.

BRIANO, L. y SAAVEDRA, M. (2015) . La composición del consejo de administración y la estructura accionaria como factores explicativos de la transparencia en el gobierno corporativo en Latinoamérica: evidencia en empresas cotizadas de Argentina, Brasil, Chile y México, Revista Estudios Gerenciales, 31 (2015) 275-286.

Bustos, O. (2002). Mujeres rompiendo el techo de cristal: el caso de las universidades. OMNIA - Revista de la Coordinación de Estudios de Posgrado(41), 1-7.

Camarena, M. y SaAvedra, M. (2016). Un estudio de las PYME lideradas por mujeres en Latinoamérica. Revista Universitaria RUTA, 18 (1) 1 27

_-Ducloux, D. (2015). Panorama del género en -México: Situación actual. Revista cientifica Guillermo de Ockham 13 (2)77-87.

Castaño, C.; Martín, J.; Vázquez, S. y Martínez, J. (2010). Female executives and the glass ceiling in Spain. International Labour Review,149 (3) 343-360.

Centro de Investigación de la Mujer en la Alta Dirección. (2013). Estadísticas sobre mujeres y empresarias en México. México. 
CUADRADO, I. y MORALES, J. (2007). Algunas claves sobre el techo de cristal en las organizaciones. Psicología de Trabajo y de las organizaciones, 23 (2), 183-202.

ChÁvez, N. y Ríos, H. (2014). Discriminación salarial por género "Efecto techo de cristal". Caso: siete áreas metropolitanas de Colombia. Dimensión Empresarial, 12 (2) 19-45.

De Anca, C. y Aragón, S. (2007). La mujer directiva en España: catalizadores e inhibidores en las decisiones de trayectoria profesional. Revista Latinoamericana de Administración(38), 45-63.

FORBES. (2014). 50 Mujeres + poderosas de México. Forbes México, 128-140.

Graglia, D. (2014). Las 500 empresas más importantes de México. Expansión, 121-248.

GurRíA, A. (25 de enero de 2013). Gender Dynamics: How Can ountries Close the Economic Gender Gap? Davos, Suiza, disponible en: http://www.oecd.org/economy/ enderdynamicshowcancountriesclosetheeconomicgendergap.htm, consultado el 25 de enero de 2017.

HernáNDEZ, E. (2010). Nuevas tendencias en el mundo empresarial: la participación de las mujeres. La ventana (32), 52-79.

Hernández, C., Camarena, M., y Castanedo, B. (2009). Para las mujeres no todo el poder pero sí todo el trabajo. México: UNAM FCA, EON.

Instituto Nacional de Estadística y Geografía (2013). Estadísticas a propósito del día internacional de la mujer. México.

- (2014). Encuesta Nacional de Empleo. México: INEGI.

Instituto Nacional de las mujeres. (2014). Empleadoras. Encuesta $\mathrm{Na}$ cional de Ocupación y empleo. México: Inmujeres. 
KAUFMANN, E. (2009). El desarrollo de la carrera de la mujer en la banca: reflexiones sobre el techo de cristal en el sector financiero. Harvard Deusto Business Review, 2-9.

MarTín, M. (2007). La mujer en al industria publicitaria. La segregación vertical en al comunicación comercial: techo de cristal y suelo pegajoso. Zer(22), 429-452.

MartíneZ, G. (2004). Empresarias y ejecutivas: referencias organizacionales y ejercicio de poder. Pérez Gil-Romo, S. y Ravelo B., Voces disidentes. Debates contemporáneos en los estudios de género. México: Porrúa, pp. 25-45.

Morrison, A., White, R. y Van Velsor, E. (1986). Rompiendo el techo de cristal. Reading: Addison-Wesley.

Organización Internacional del Trabajo (2012). Global Employment Trends for Women 2012. International Labour Office -Geneva: ILO.

- (2014a). Romper el techo de cristal. Las mujeres en puestos de dirección. Ginebra: OIT

— (2014b). Desarrollo de la iniciativa empresarial de la mujer. Ginebra: OIT, Programa de Empresas Sostenibles Desarrollo e iniciativa empresarial de la Mujer.

Ramos, A.; Barberá, E. y SARrió, M. (2003). Mujeres directivas, espacio de poder y relaciones de género. Anuario de Psicología, 34, (2), 267278.

RoldÁn-García, E., Leyra-FATOU, B., y CONTRERAs-MartíneZ, L. (2012). Segregación laboral y techo de cristal en el trabajo social: análisis del caso español. Redalyc.org, XII(2), 43-56. 
TARAngo, Y. (2013). Mujeres empresarias mexicanas. Obtenido de Vértigo Político: http://www.vertigopolitico.com/articulo/5832/Mujeresempresarias-mexicanas, consultado el 15 de julio de 2016.

Torres, O. y Pau, B. (2011). "Techo de cristal” y suelo pegajoso. La situación de la mujer en los sistemas alemán y español de ciencia y tecnología. Revista CTS, 6(18), 35-39.

World Economic Forum (2014). The Global Gender Gap Report 2014. Suiza: WEF.

Zabludovsky, G. (1997). Presencia de las mujeres ejecutivas en México. Sociológica(33), 73-97. 